\title{
The Effect of Vitamins B on Improving the Symptoms of Migraine: An Overview
}

\section{Shiva Nematgorgani ${ }^{1}$, Zahra Gholi ${ }^{1}$, Soodeh Razeghi Jahromi ${ }^{1}$, Mansoureh Togha ${ }^{2 *}$ Fariba Karimzadeh $^{3 *}$}

${ }^{1}$ Department of Clinical Nutrition and Dietetics, Faculty of Nutrition and Food Technology, Shahid Beheshti University of Medical Sciences, Tehran, Iran

${ }^{2}$ Iranian Center of Neurological Research, Neuroscience Institute, Tehran University of Medical Sciences, Tehran, Iran

${ }^{3}$ Cellular and Molecular Research Center, Iran University of Medical Sciences, Tehran, Iran

\section{ABSTRACT}

Introduction: Migraine is a common, painful and potentially debilitating disease that is characterized by frequent, unilateral, pulsating attacks of moderate to severe severity. The main cause of migraine is unknown. It seems the B vitamins affect clinical symptoms of the migraine headache. In this study, we reviewed the effects of some vitamins B on improving the symptoms of migraine headaches and their possible mechanism of action. In this study, we evaluated the articles published between 2002 and 2018 on this topic in Scopus, Science Direct, PubMed, and Google Scholar. Conclusion: The findings of several studies suggest that some of vitamins B can improve migraine headaches, particularly their frequency and severity. Dietary supplementation of vitamins B could efficiently prevent or alleviate different symptoms of the migraine headache.

*Corresponding Authors: Mansoureh Togha, Fariba Karimzadeh

E-mail: toghae@sina.tums.ac.ir,kaimzade.f@iums.ac.ir

Key words:

1. Migraine Disorders

2. Thiamine

3. Folic Acid

4. Pyridoxine

5. Vitamin B 12 
تأثير ويتامينهاى گروه B بر بهبود علايم بيمارى ميخرن: يك مقاله مرورى

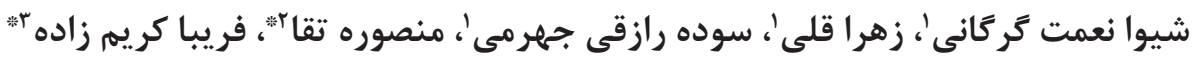

$$
\begin{aligned}
& \text { كروه تغذيه بالينى و رزيم درمانى، دانشكده علوم تغذيه و صنايع غذايى، دانشخاه علوم يزشكى شهيد بهشتى، تهران، ايران } \\
& \text { 'مركز تحقيقات علوم اعصاب ايران، يزوهشكده علوم اعصاب، دانشخاه علوم يزشكى تهران، تهران، ايران } \\
& \text { "مركز تحقيقات سلولى و مولكولى، دانشخاه علوم يزشكى ايران، تهران، ايران يرومان }
\end{aligned}
$$

\section{اطلاعات مقاله:}

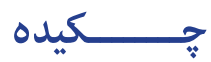

مقدمه: ميكرن يك بيمارى شايع، دردناك و به طور بالقوه ناتوان كننده است كه با حملات مكرر، يكطرفه

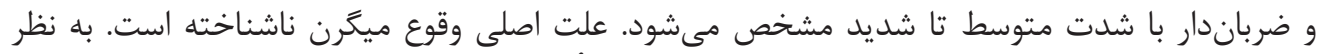

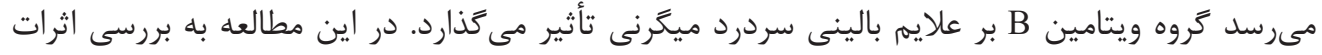

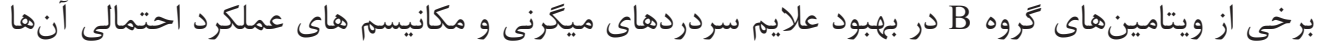

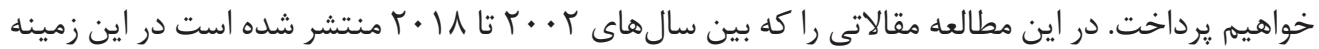

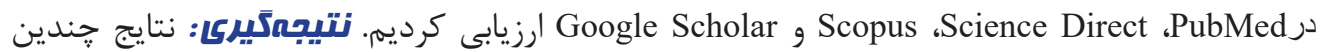

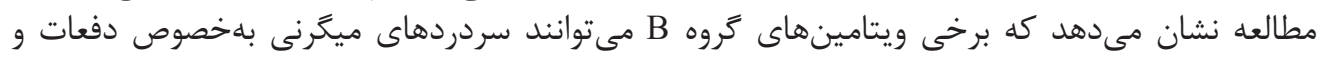

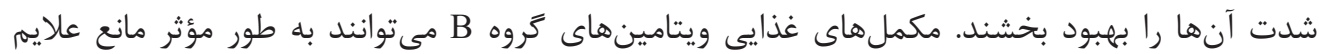
مختلف سردرد ميكرنى و يا كاهش آن آن شوند.

كليد وازمها:

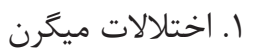

r. r. r r

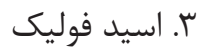

f

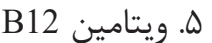

* نويسند " آدرس الكترونيكى: toghae@sina.tums.ac.ir, kaimzade.f@iums.ac.ir 


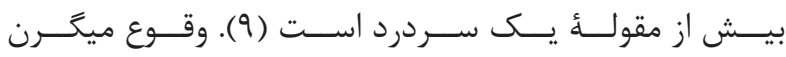

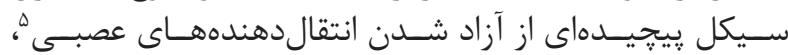

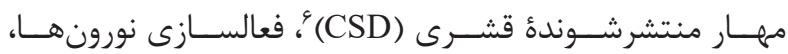

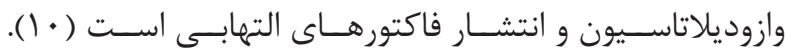

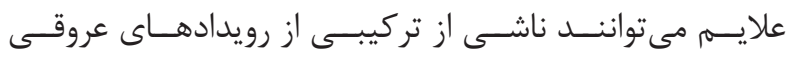

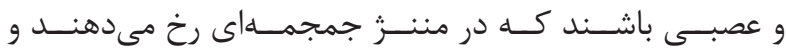

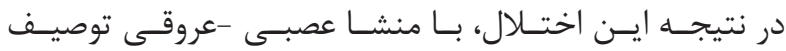

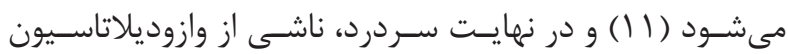

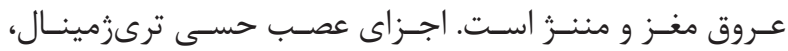

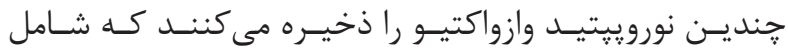

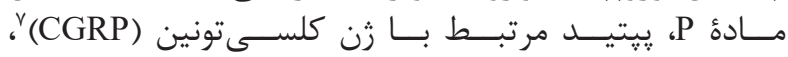

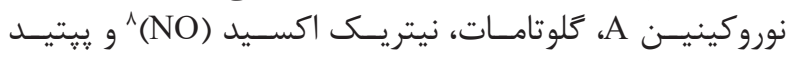

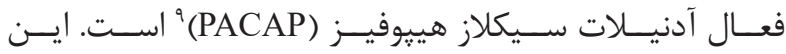

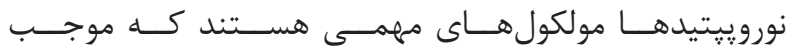

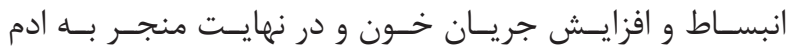

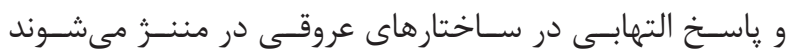

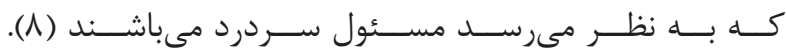

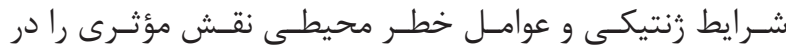

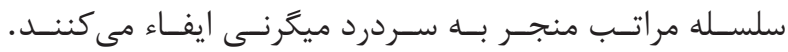

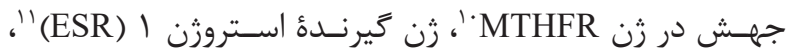

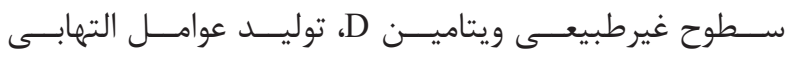

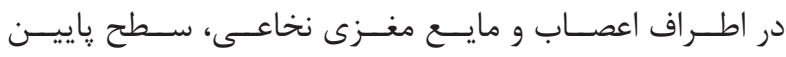

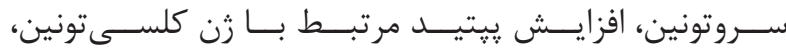

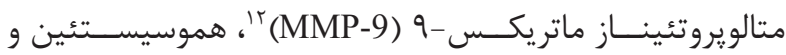

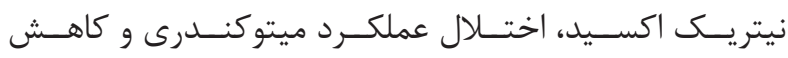

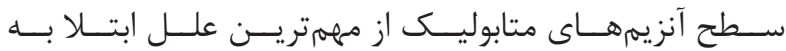

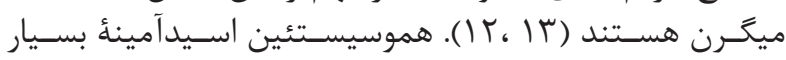

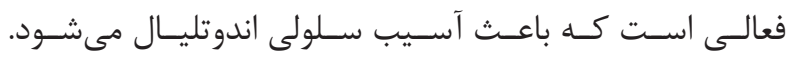

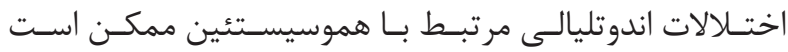

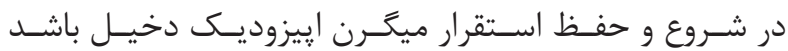

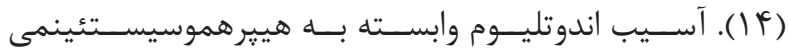

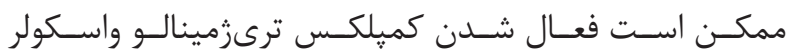

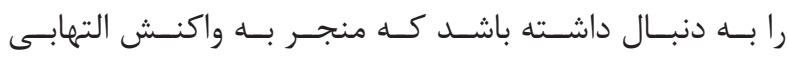

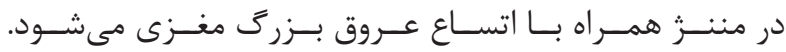

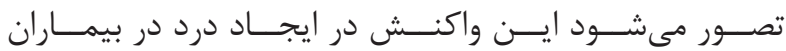

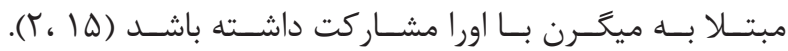

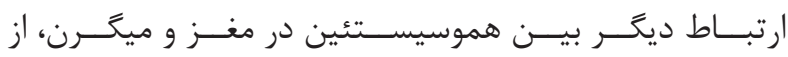

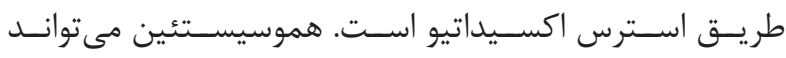

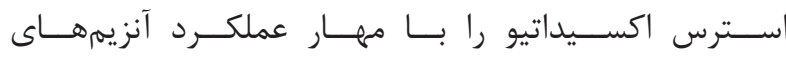

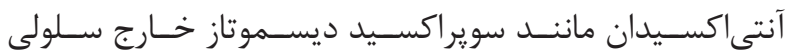

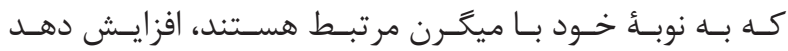

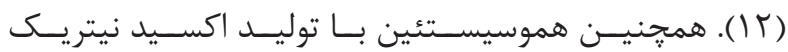

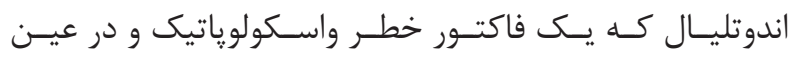

${ }^{1}$ World health organization

${ }^{2}$ International classification of headache disorders

${ }^{3}$ International headache society

${ }^{4}$ Migraine with aura

${ }^{5}$ Neurotransmitters

${ }^{6}$ Cortical spreading depression

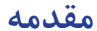

ميخــرن يـــ بيمـارى شـايع، دردنــاك و در مــوارد شــديد و

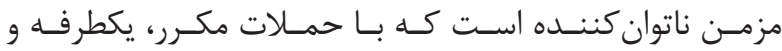

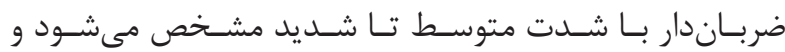

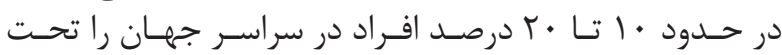

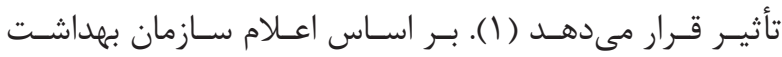

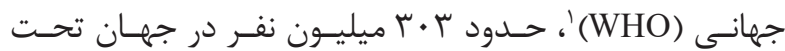

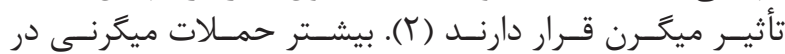

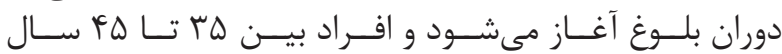

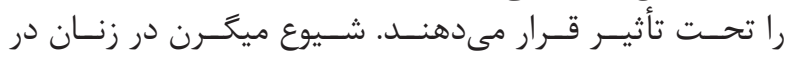

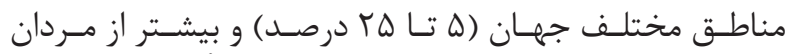

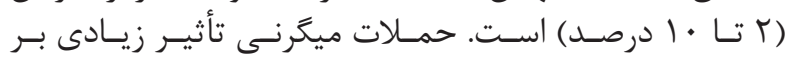

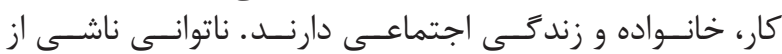

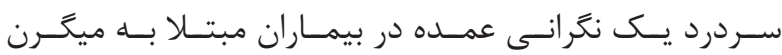

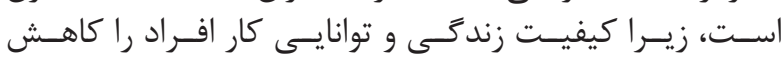

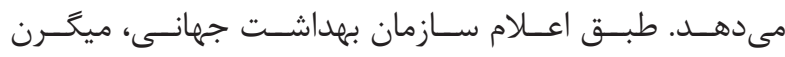

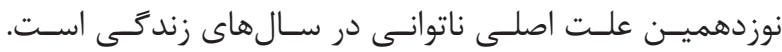

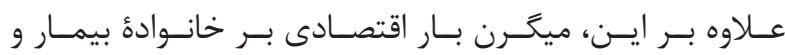

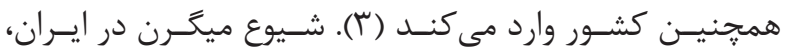

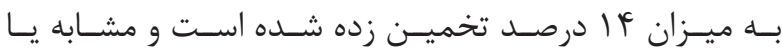

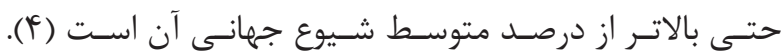

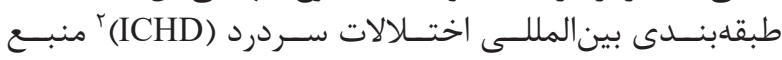

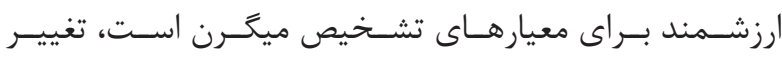

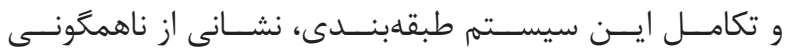

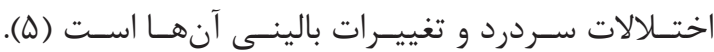

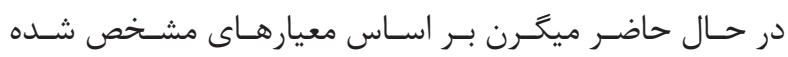

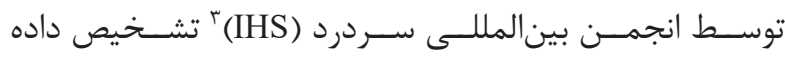

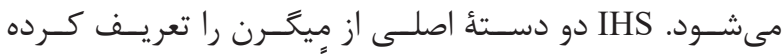

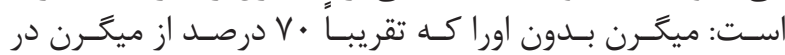

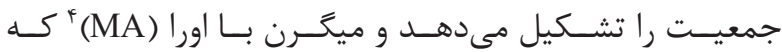

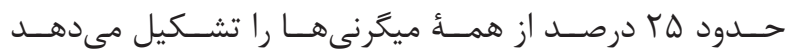

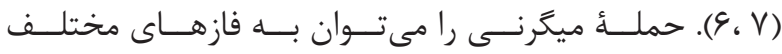

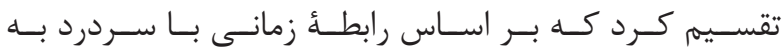

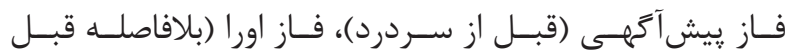

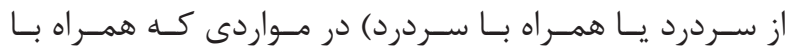

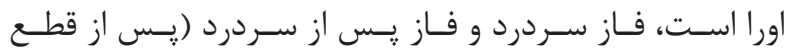

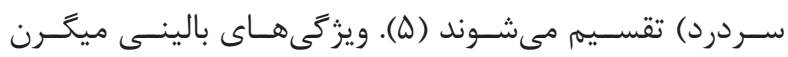

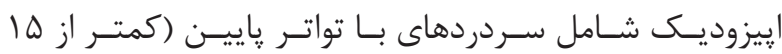

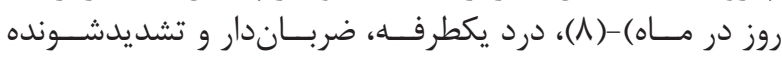

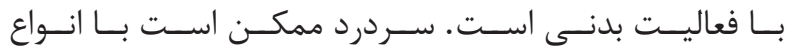

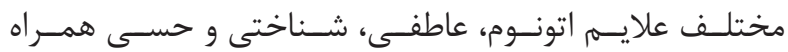

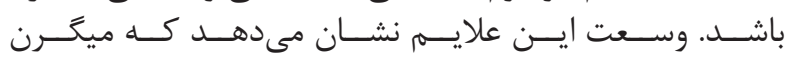

${ }^{7}$ Calcitonin gene-related peptide

${ }^{8}$ Nitric oxide

${ }^{9}$ Pituitary adenylyl cyclase activating polypeptide

${ }^{10}$ Methylene tetrahydrofolate reductase

${ }^{11}$ Erythrocyte sedimentation rate

${ }^{12}$ Matrix metalloproteinase 9 


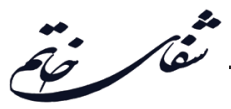

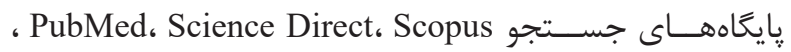
جمـع آورى و بررسـى شـــ بـراى جسـتجو ، Google Scholar از كليـد وازههــاى Bitamin ،headache ، migraine disorders ،folic acid ،vitamin B12 ، vitamin B9 ،vitamin B1 36 (pyridoxine ، thiamine ،cobalamin

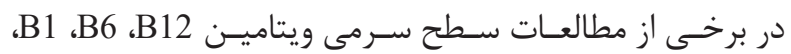

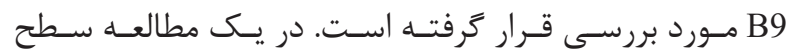

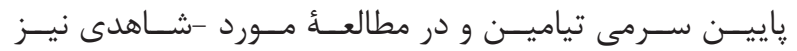

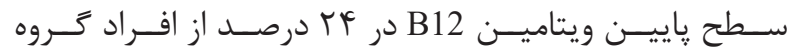

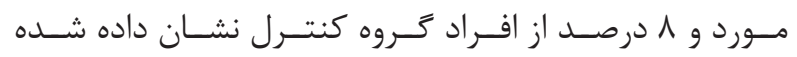

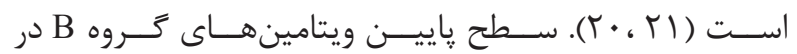

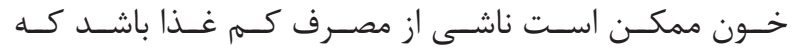

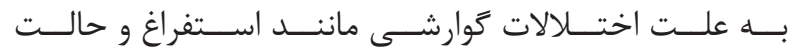

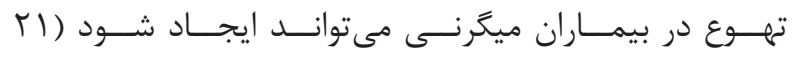

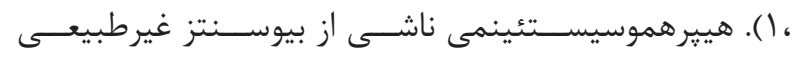

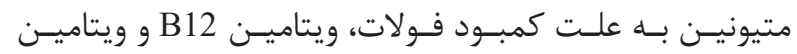

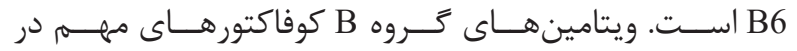

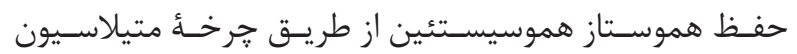

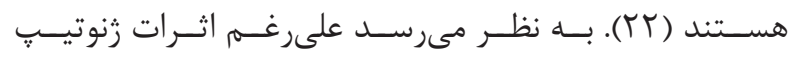

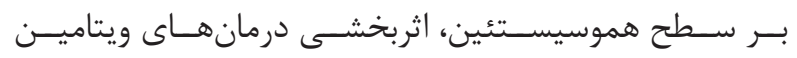

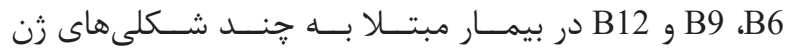

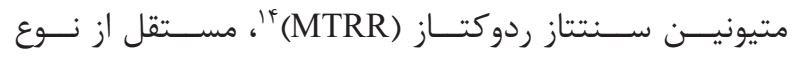

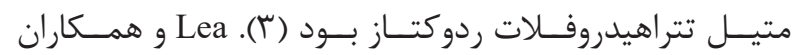

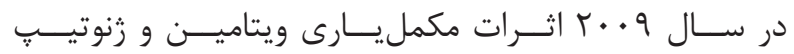

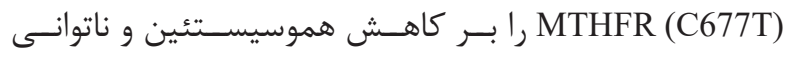

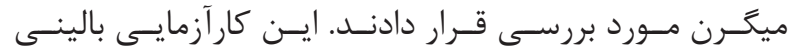

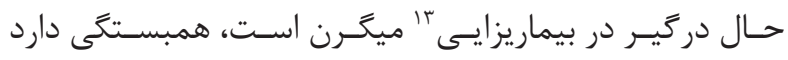

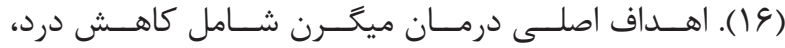

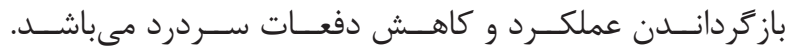

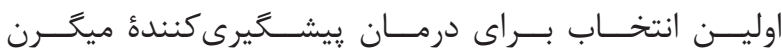

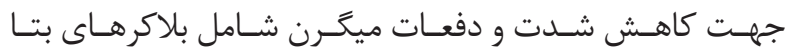

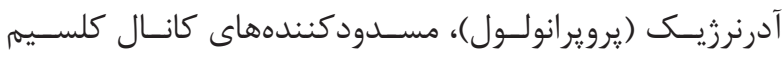

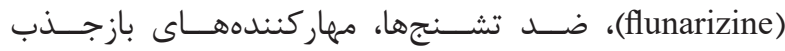

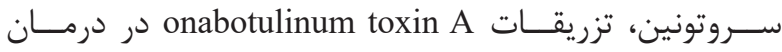

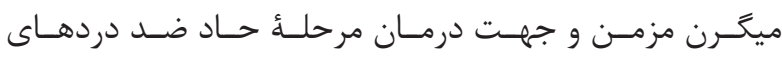

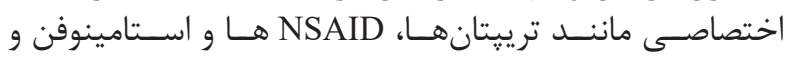

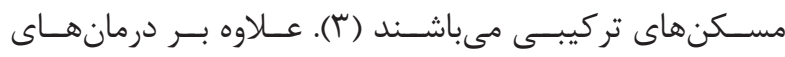

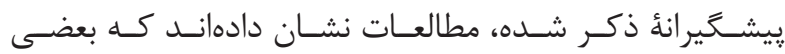

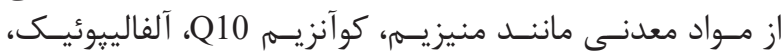

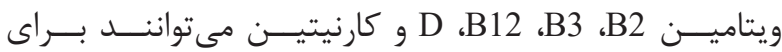

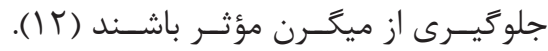

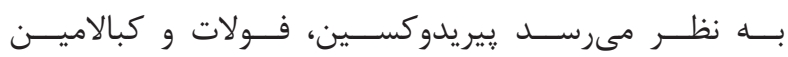

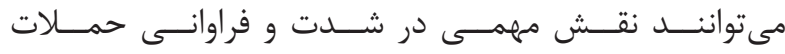

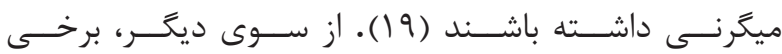

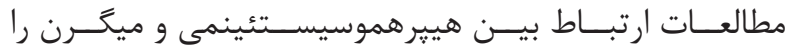

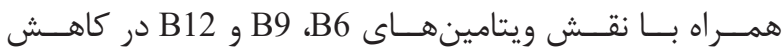

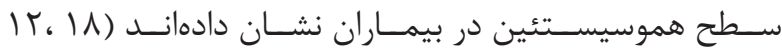

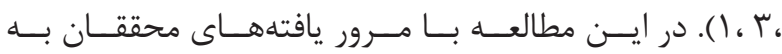

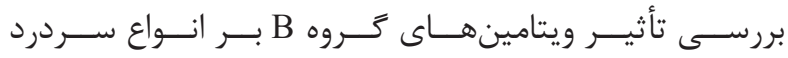

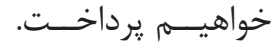

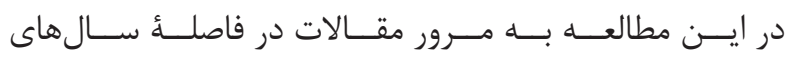

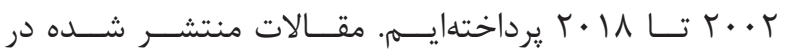

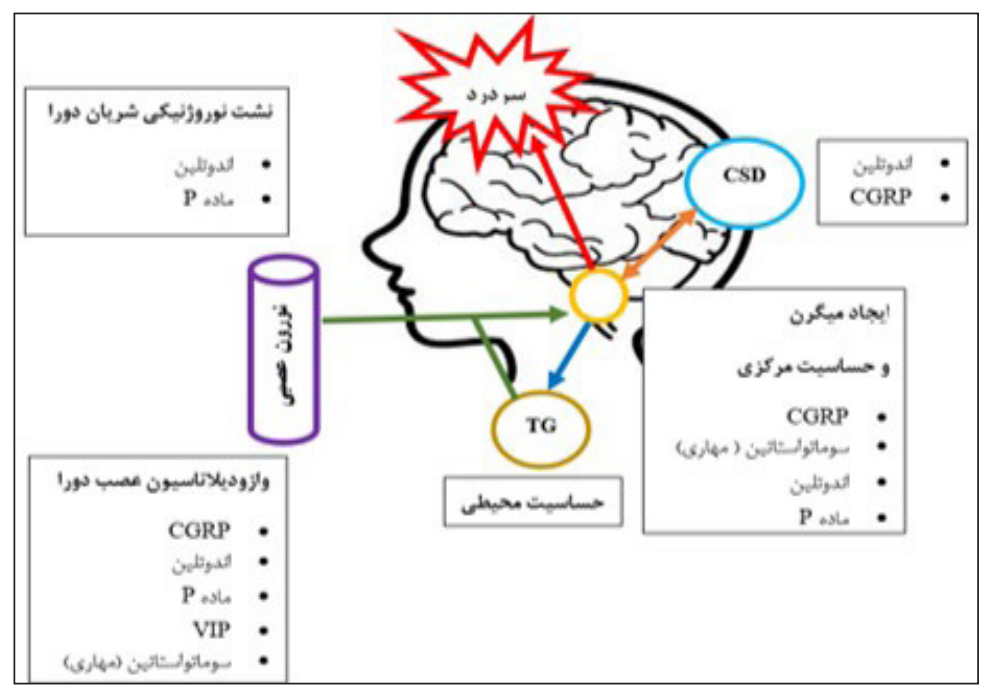

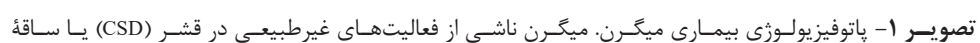

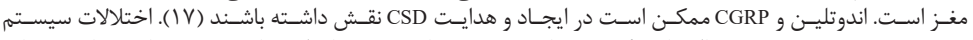

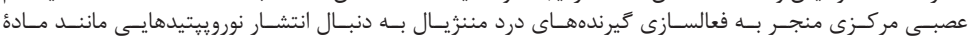

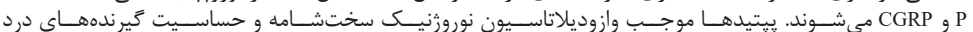

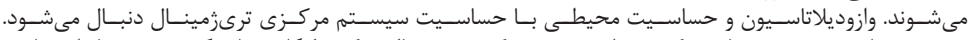

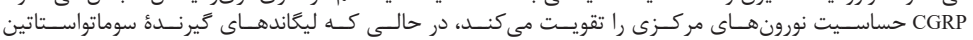

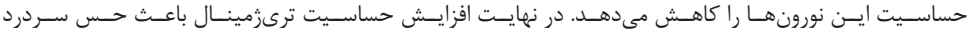

\footnotetext{
${ }^{13}$ Pathogenicity

${ }^{14}$ Methionine synthase reductase
} 


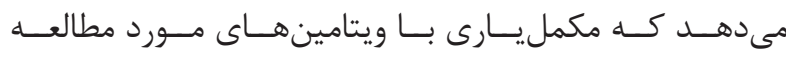

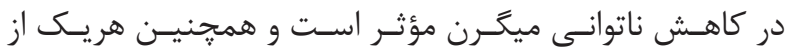

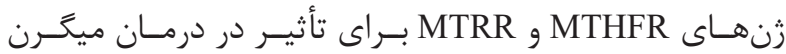

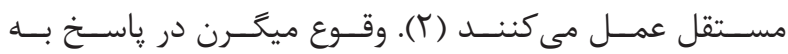

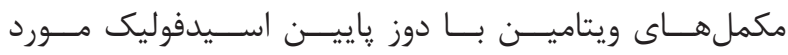

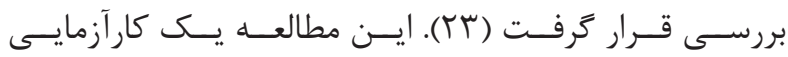

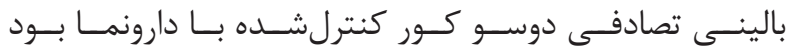

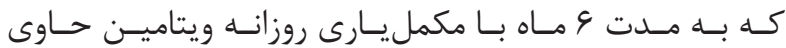

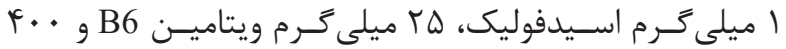

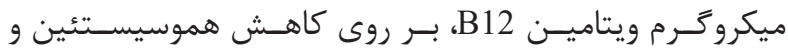

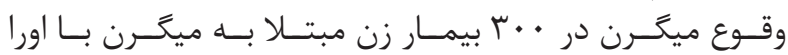

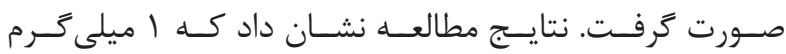

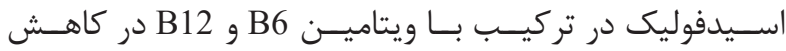

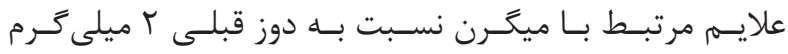

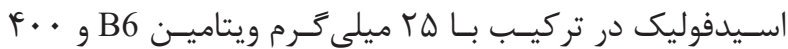

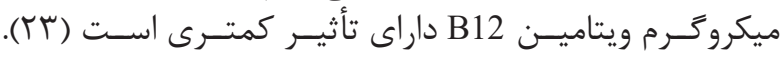

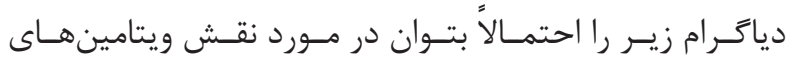

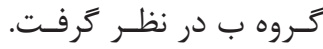

ويتامين هاى

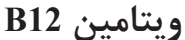

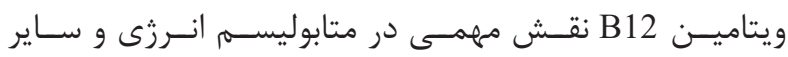

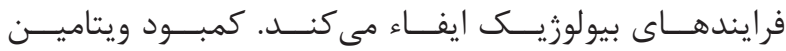

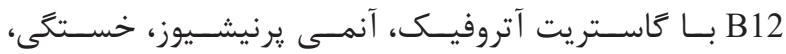

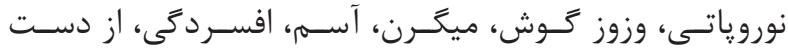

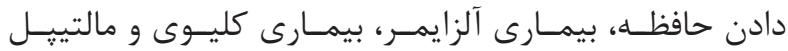

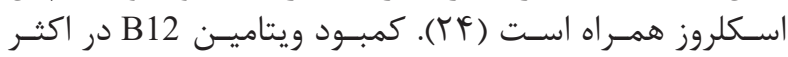

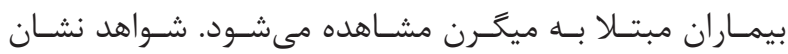

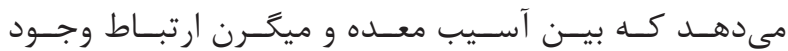

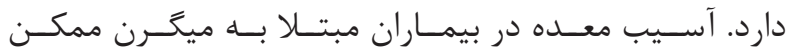

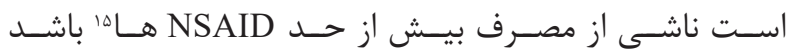

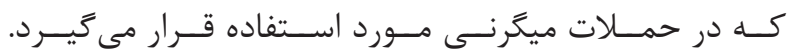

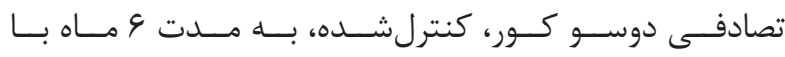

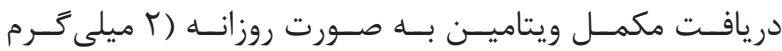

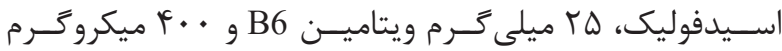

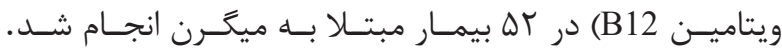

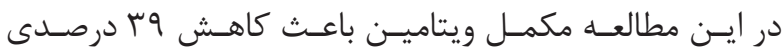

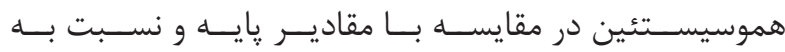

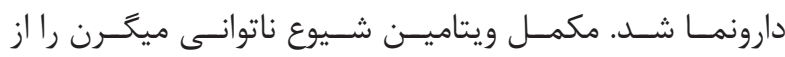

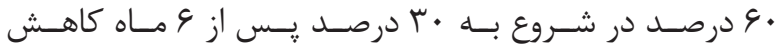

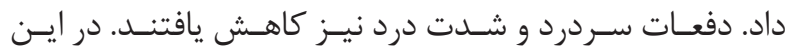

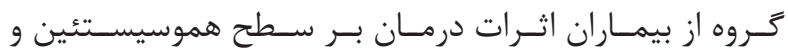

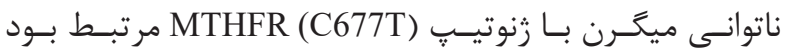

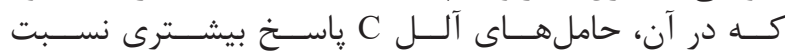

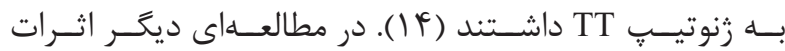

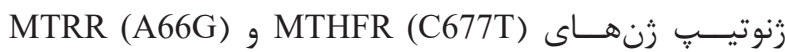

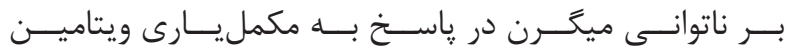

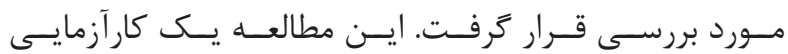

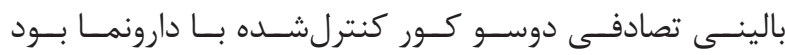

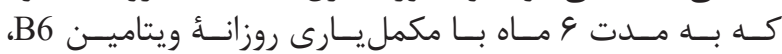

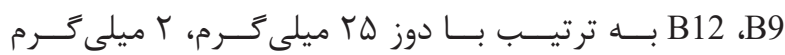

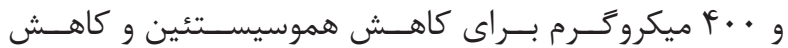

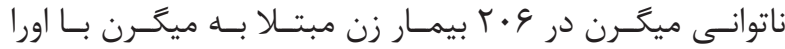

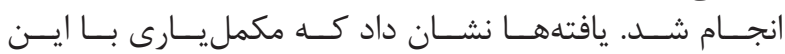

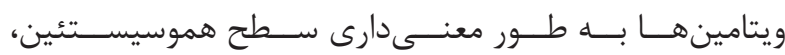

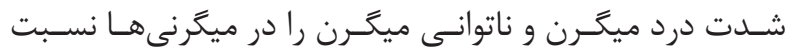

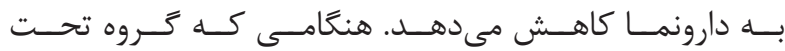

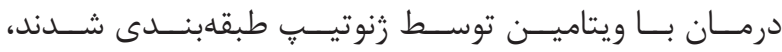

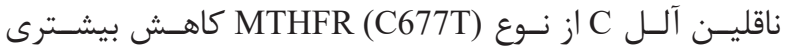

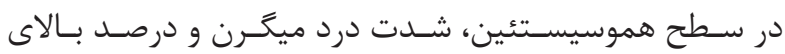

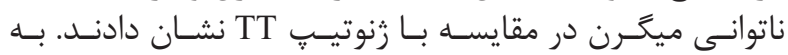

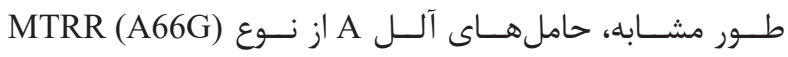

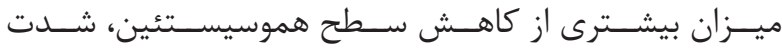

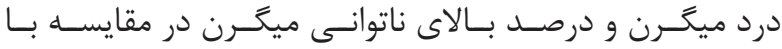

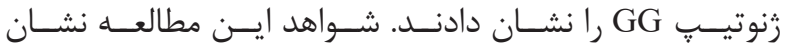

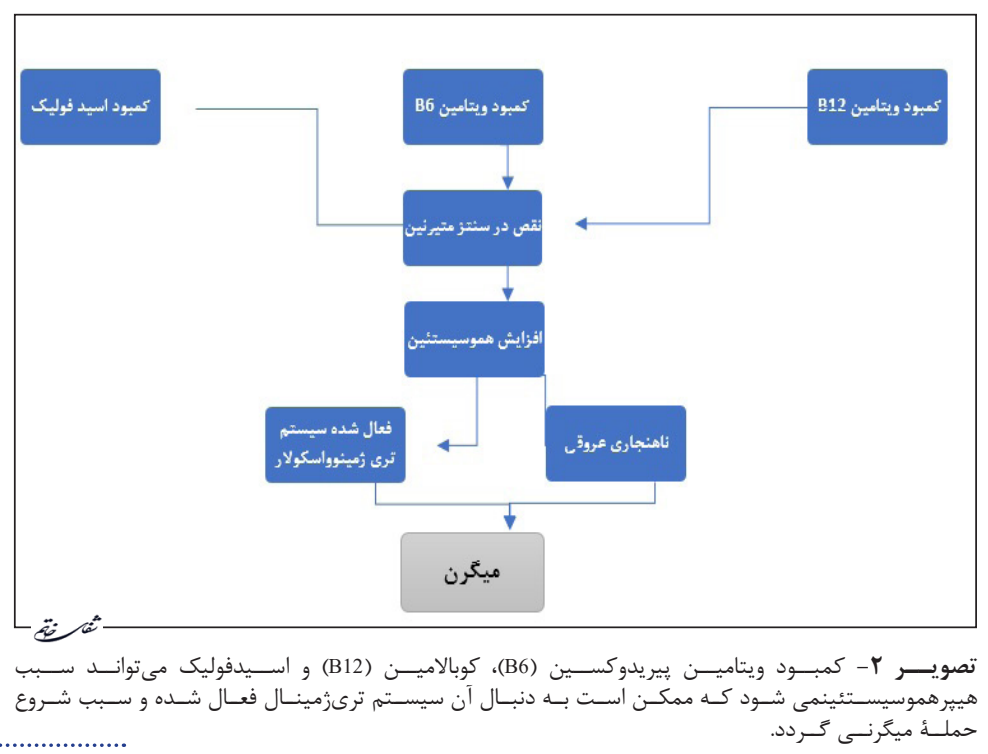

${ }^{15}$ Nonsteroidal anti-inflammatory drugs 


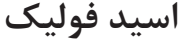

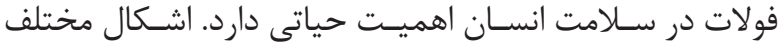

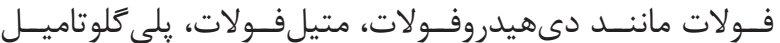

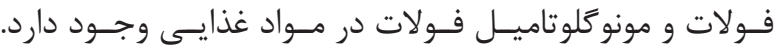

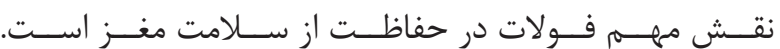

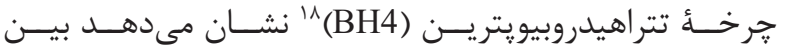

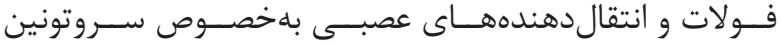

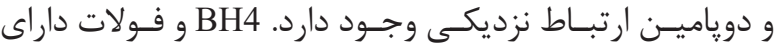

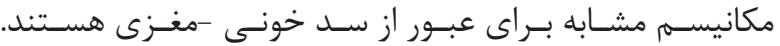

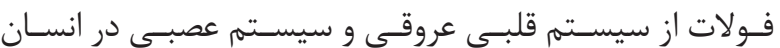

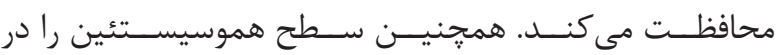

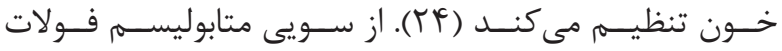

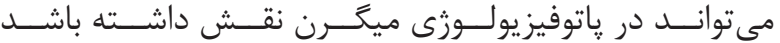

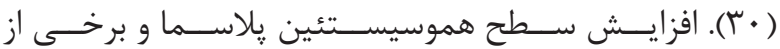

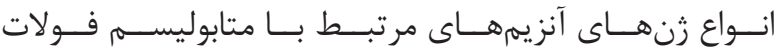

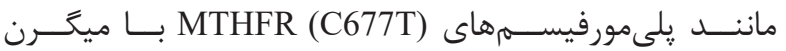

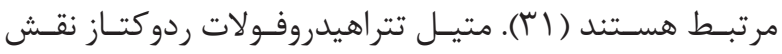

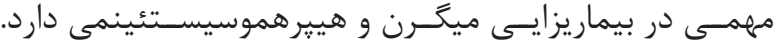

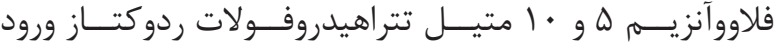

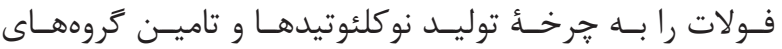

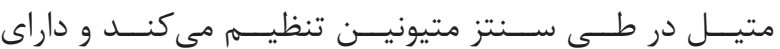

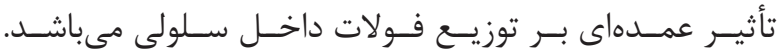

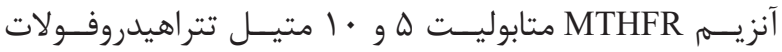

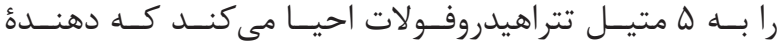

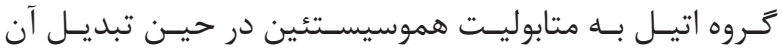

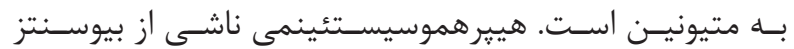

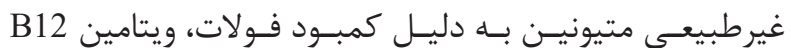

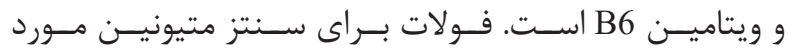

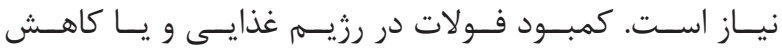

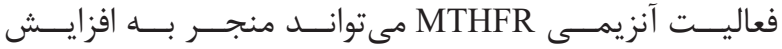

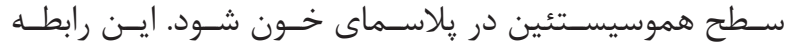

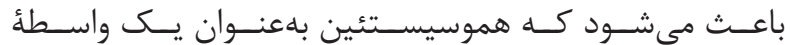

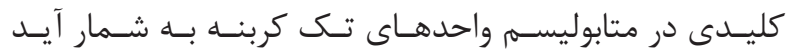

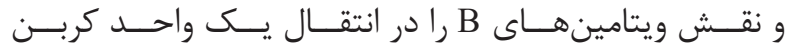

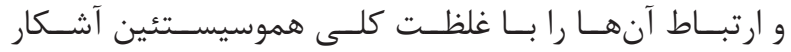

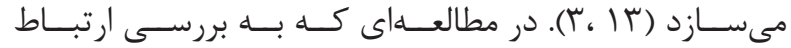

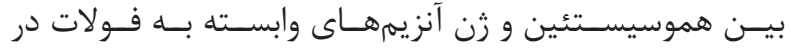

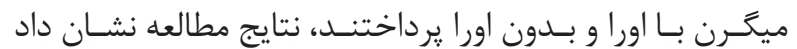

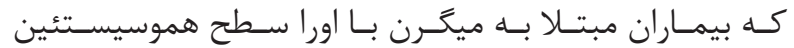

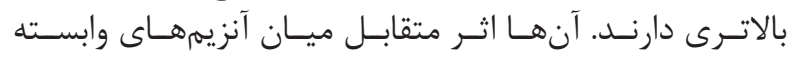

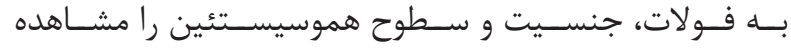

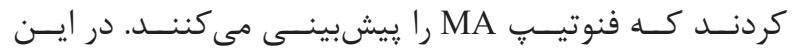

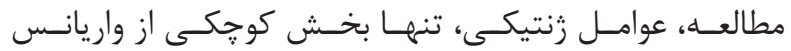

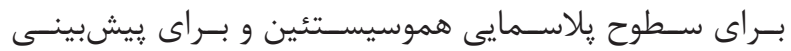

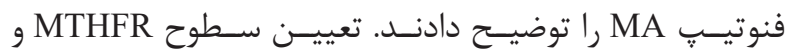

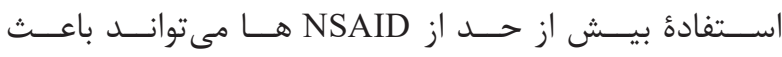

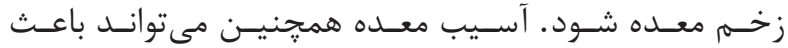

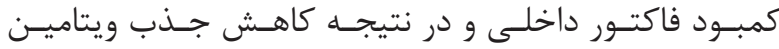

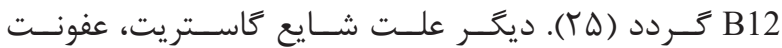

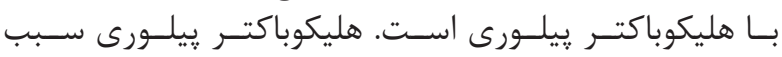

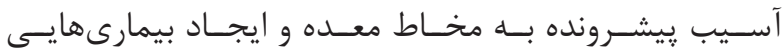

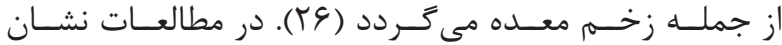

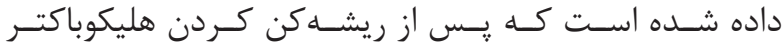

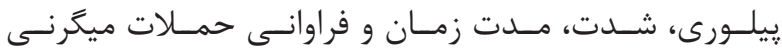

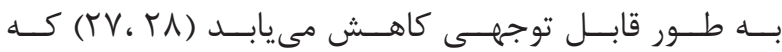

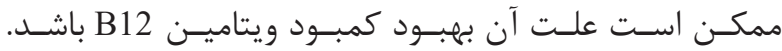

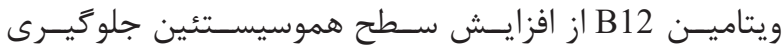

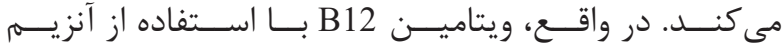

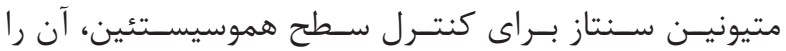

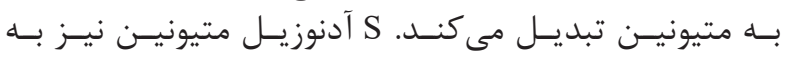

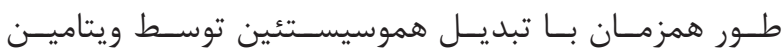

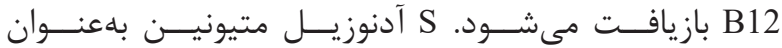

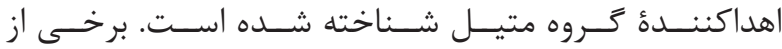

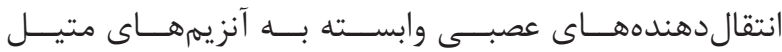

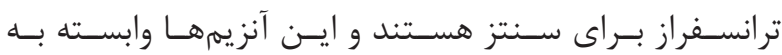

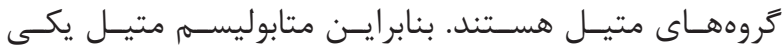

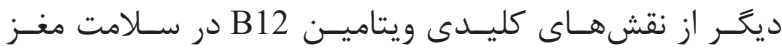

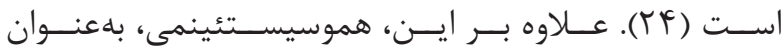

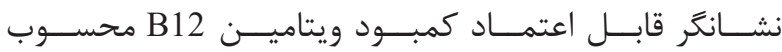

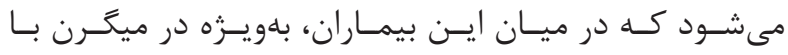

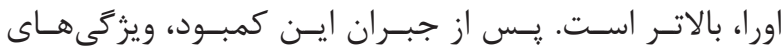

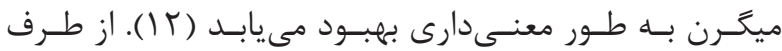

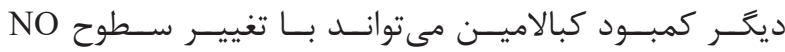

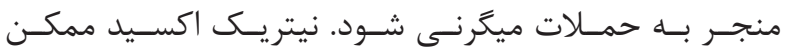

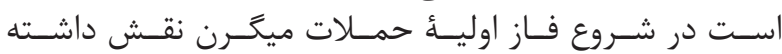

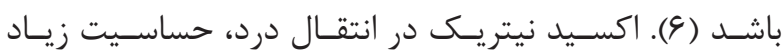

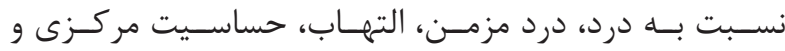

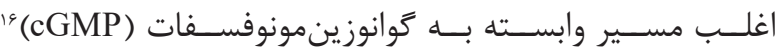

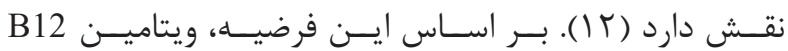

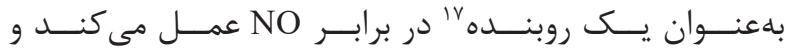

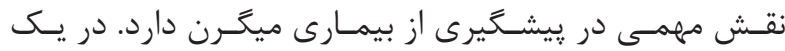

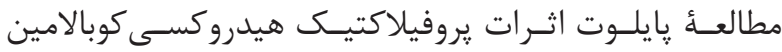

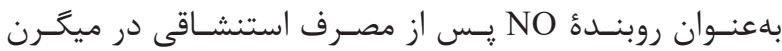

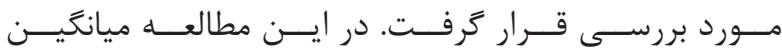

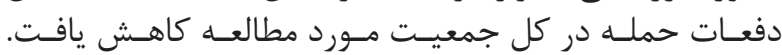

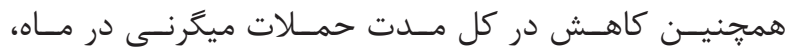

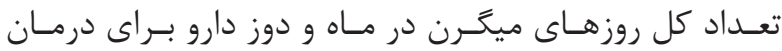

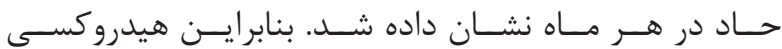

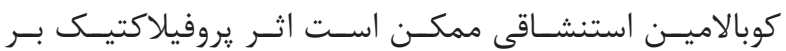

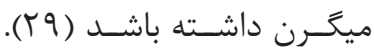

\footnotetext{
${ }^{16}$ Cyclic guanosine monophosphate

${ }^{17}$ Scavenger

${ }^{18}$ Tetrahydrobiopterin
} 


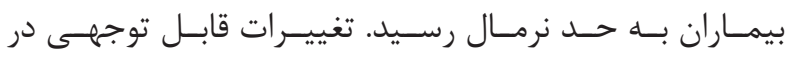

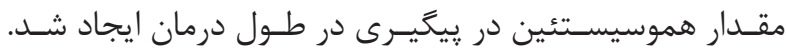

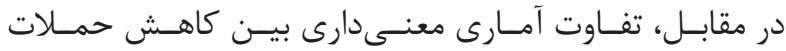

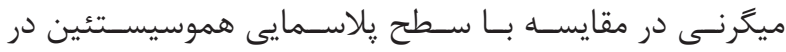

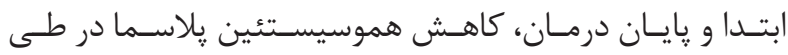

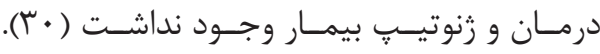

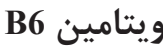

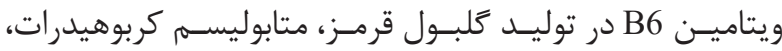

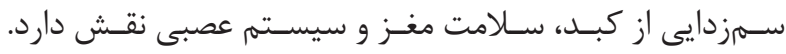

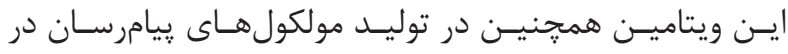

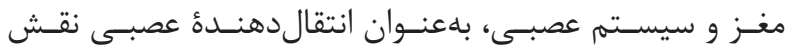

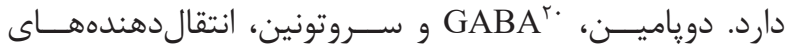

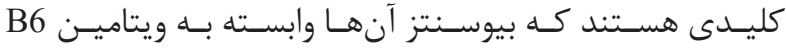

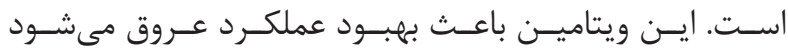

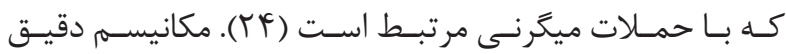

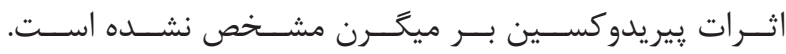

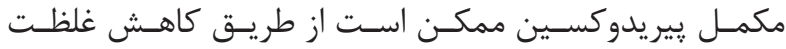

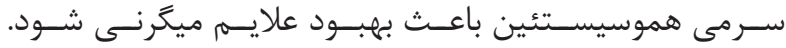

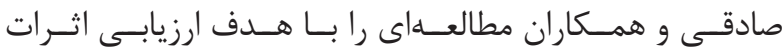

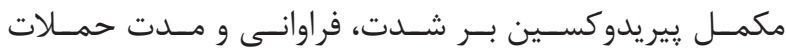

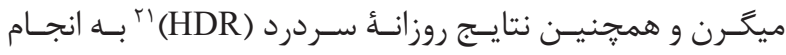

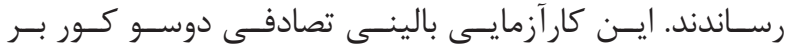

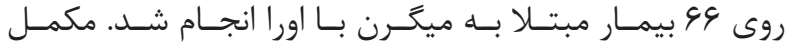

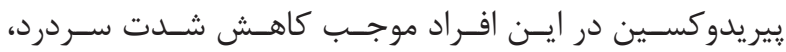

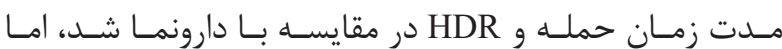

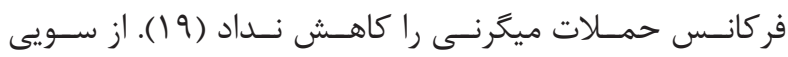

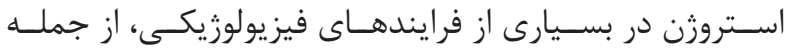

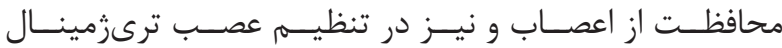

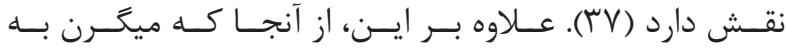

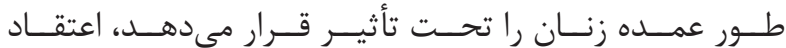

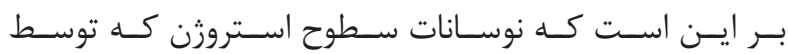

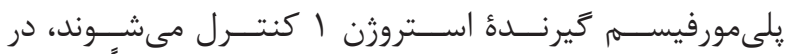

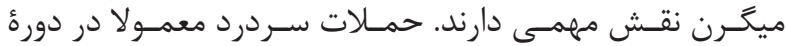

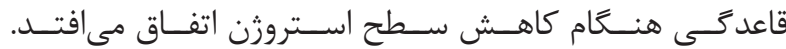

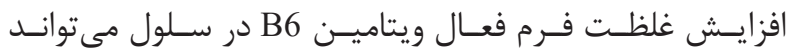

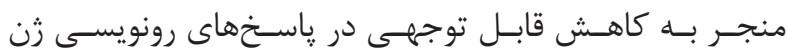

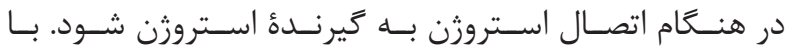

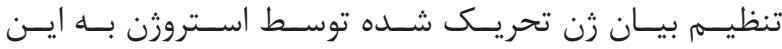

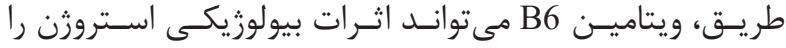

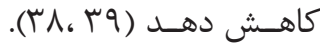

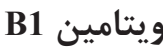

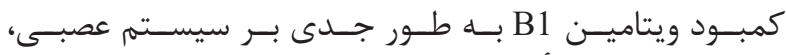

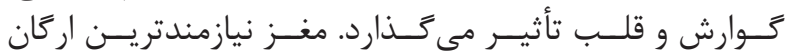

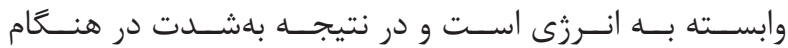

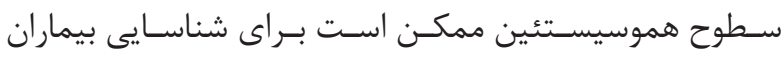

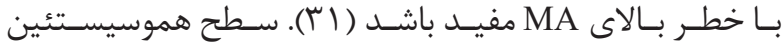

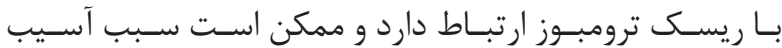

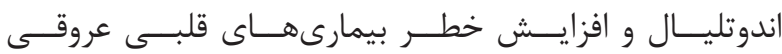

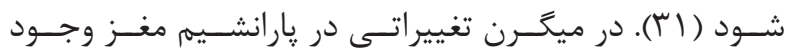

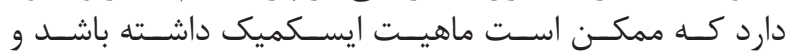

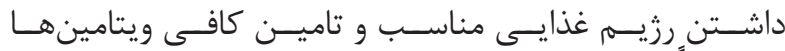

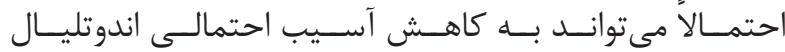

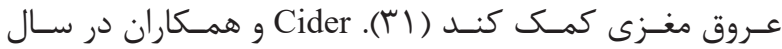

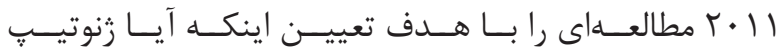

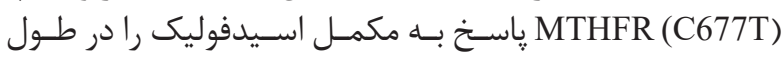

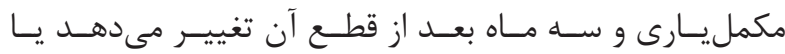

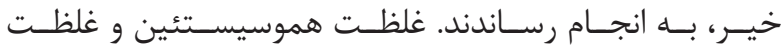

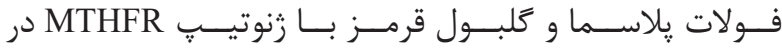

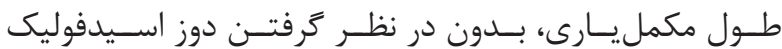

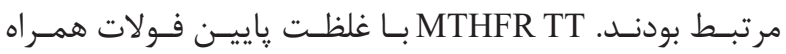

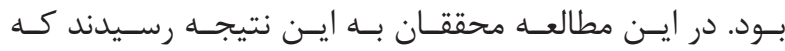

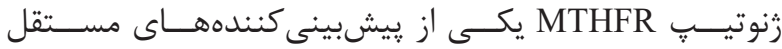

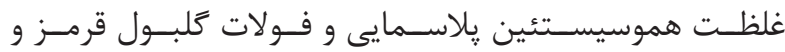

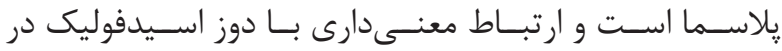

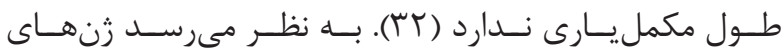

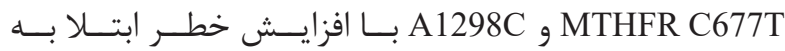

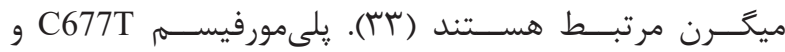

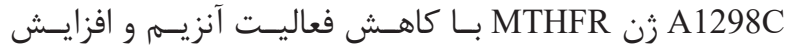

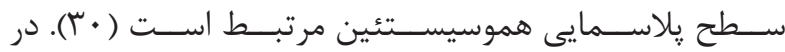

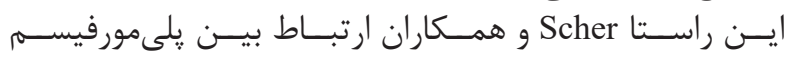

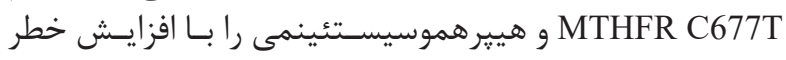

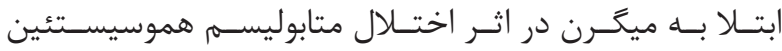

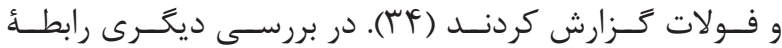

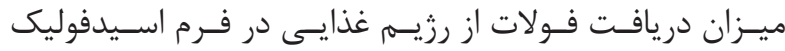

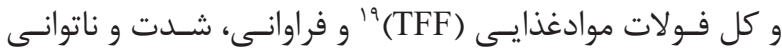

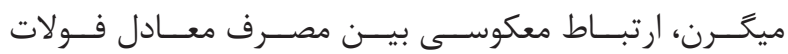

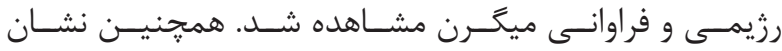

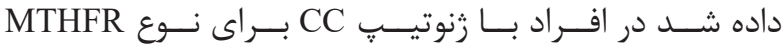

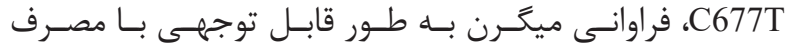

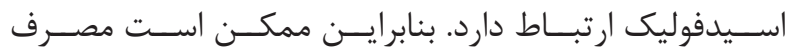

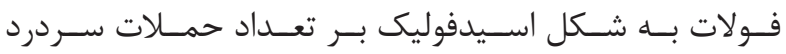

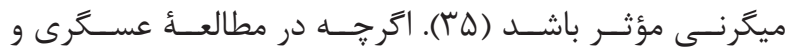

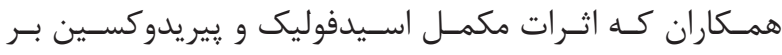

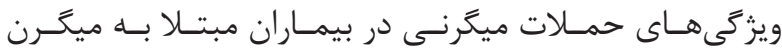

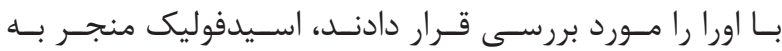

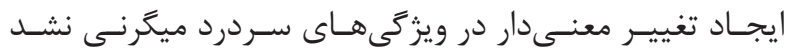

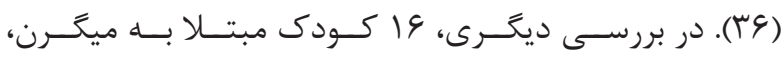

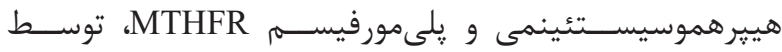

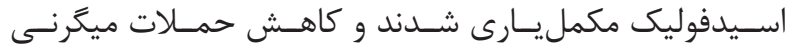

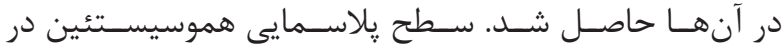

${ }^{19}$ Total food folate

${ }^{20}$ Gamma-aminobutyric acid

${ }^{21}$ Headache diary result 


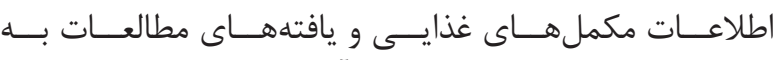

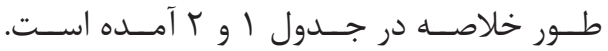
نتيجهَ كيرى

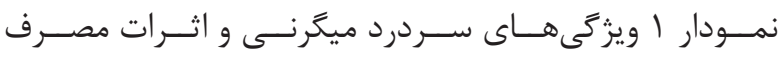

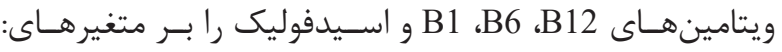

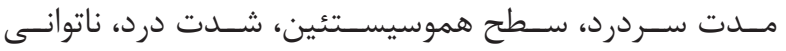

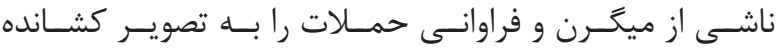

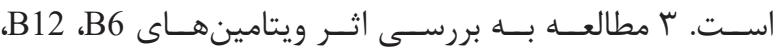

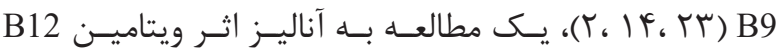

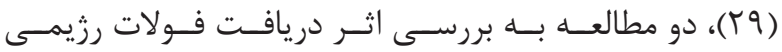

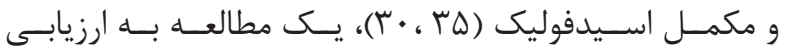

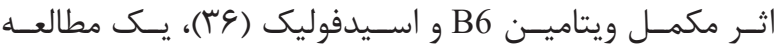

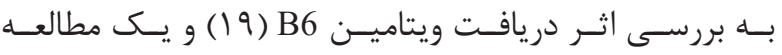

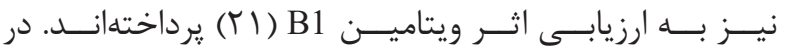

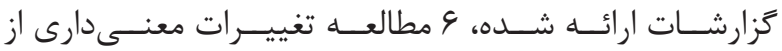

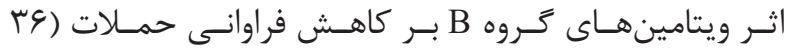

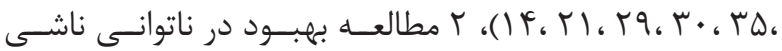

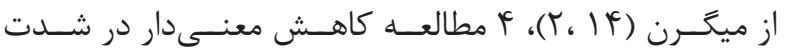

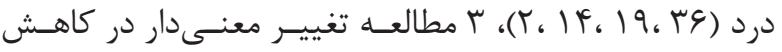

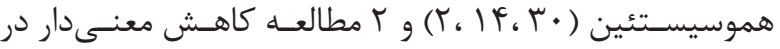

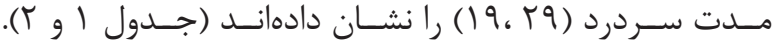

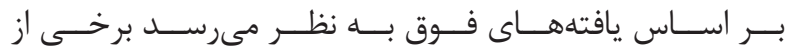

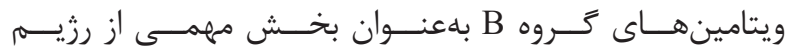

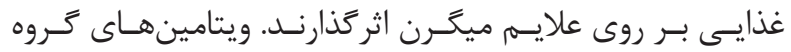

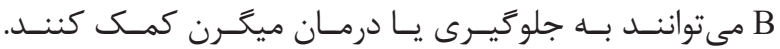

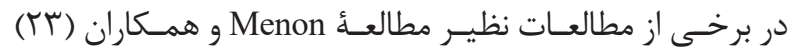

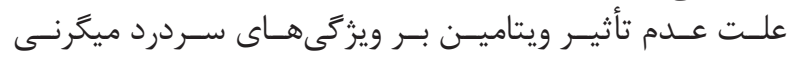

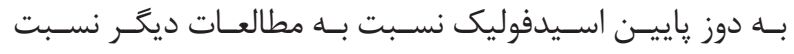

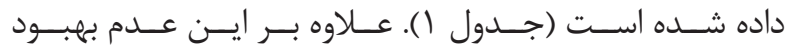

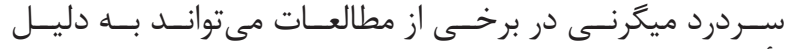

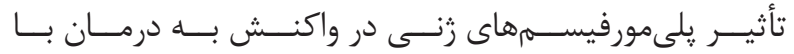

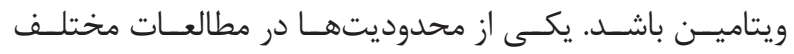

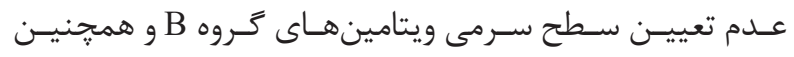

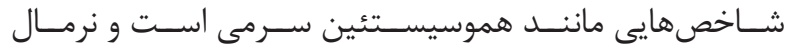

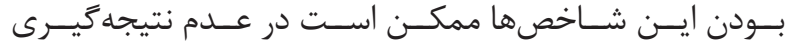

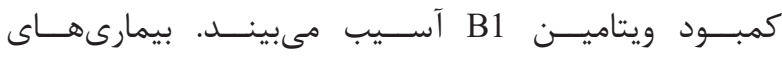

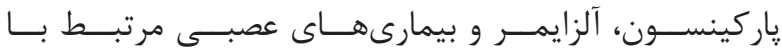

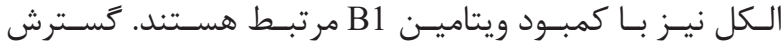

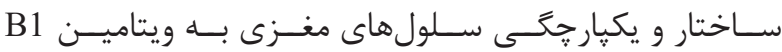

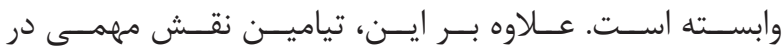

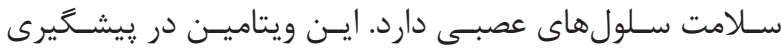

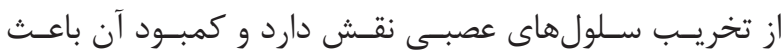

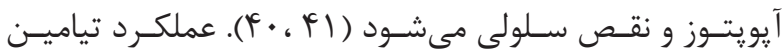

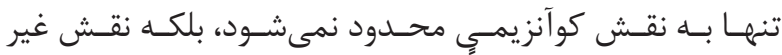

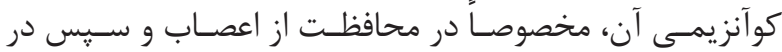

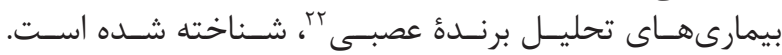

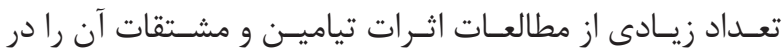

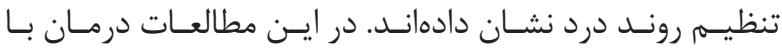

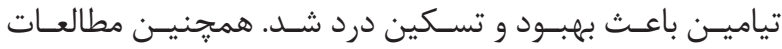

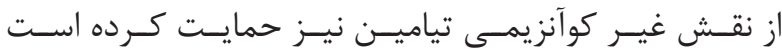

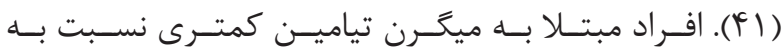

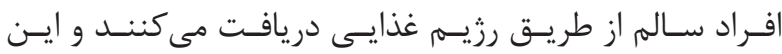

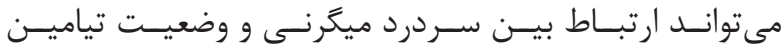

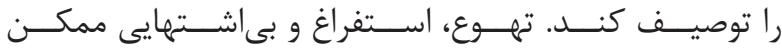

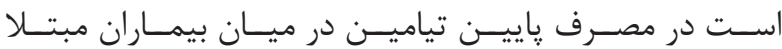

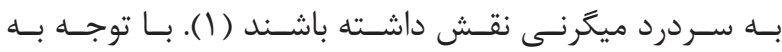

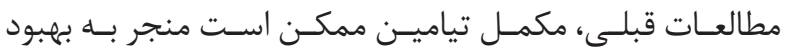

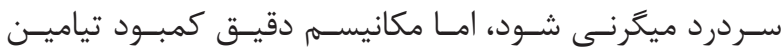

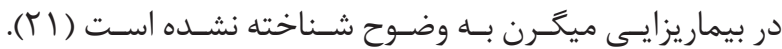

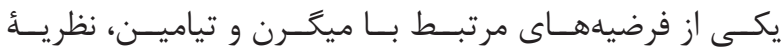

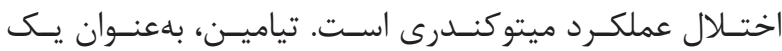

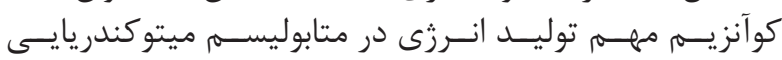

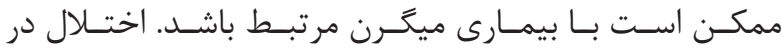

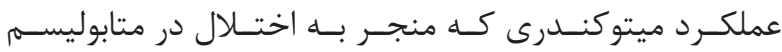

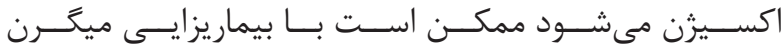

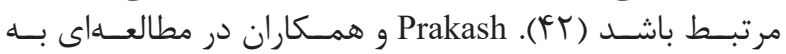

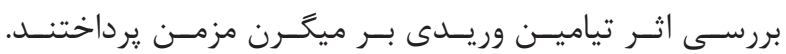

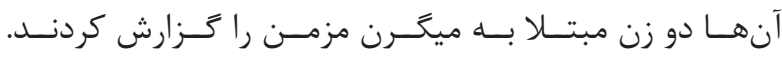

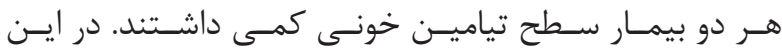

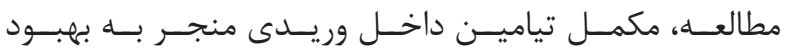

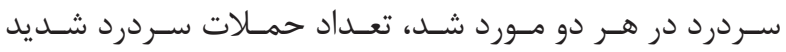

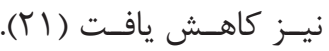

جدول ا- منابع غذايى ويتامينهاى گروه B (F (F).

\begin{tabular}{|c|c|}
\hline منبع غذايى & ويتامين \\
\hline مخمر، جكر، غلات كامل & نيامين (B1) \\
\hline كوشيتها، فرآوردمهاى غلات كامل (كمندم)، سبزيجات. مغزها، سيبزمبنى شيرين. موز & بيريدوكسين (B6) \\
\hline 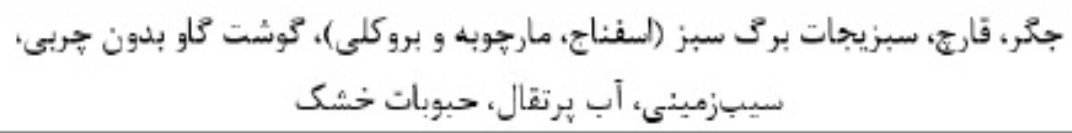 & اسيد فوليك (B9) \\
\hline 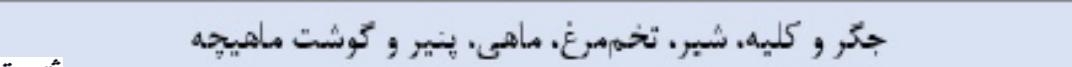 & كوبالامين (B12) \\
\hline
\end{tabular}

${ }^{22}$ Neurodegenerative 
جدول r- مرورى بر مطالعات و نتايج آنها.

\begin{tabular}{|c|c|c|c|}
\hline نتايج & نوع عدائمله & عدت مداثله. تعداد نمونه & نويسنده. سال \\
\hline 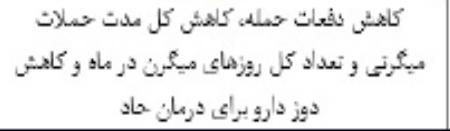 & ا ميلى كرم/روز هيدروكسى كتيا الامين & 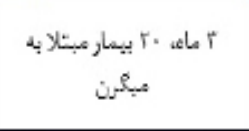 & 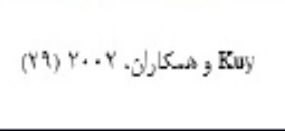 \\
\hline 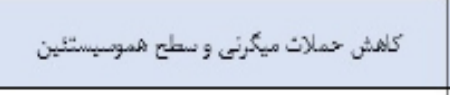 & ه هيلى كرم/روز لسيدفويك & 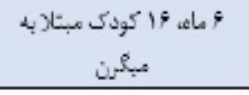 & 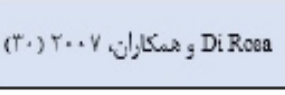 \\
\hline 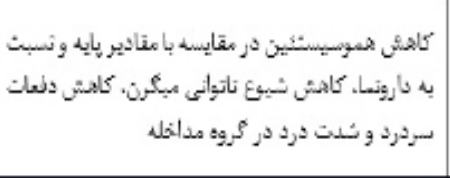 & 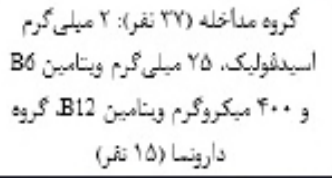 & 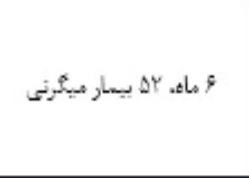 & 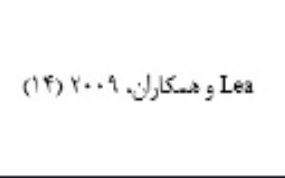 \\
\hline 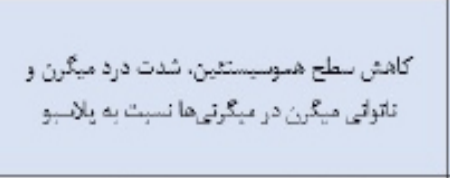 & 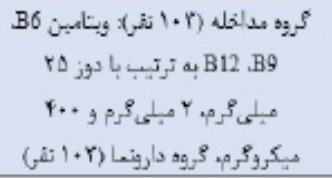 & 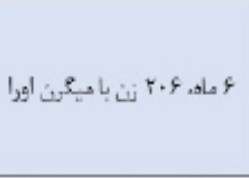 & (r) Y. M و هـكاراني. Mlenon \\
\hline 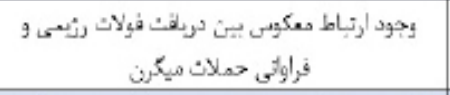 & 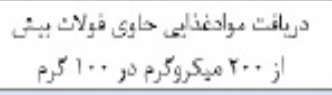 & | & 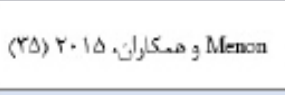 \\
\hline 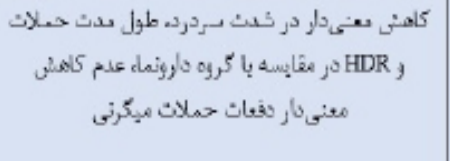 & 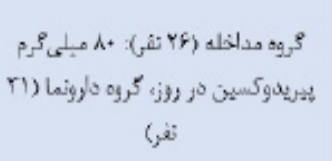 & 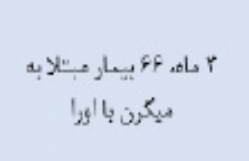 & 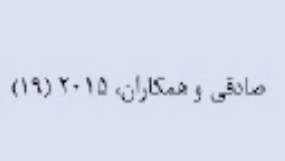 \\
\hline 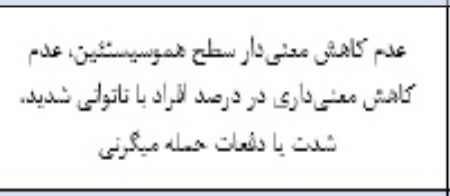 & 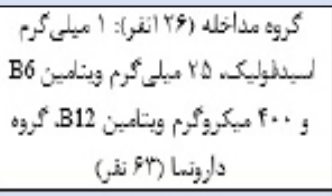 & 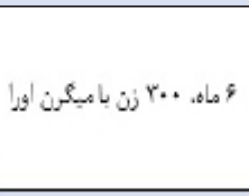 & 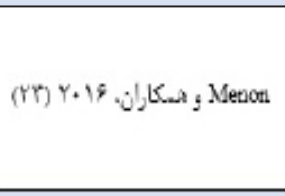 \\
\hline 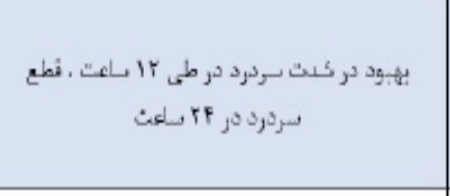 & 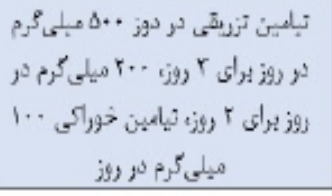 & 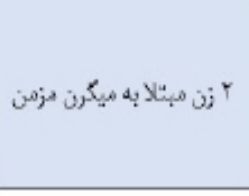 & 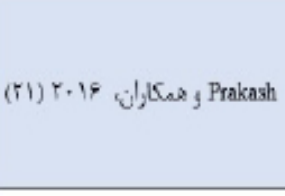 \\
\hline 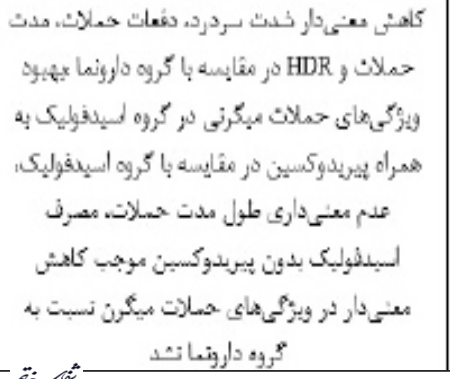 & 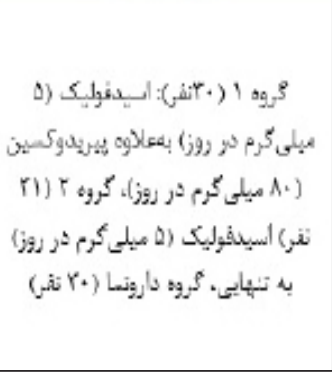 & 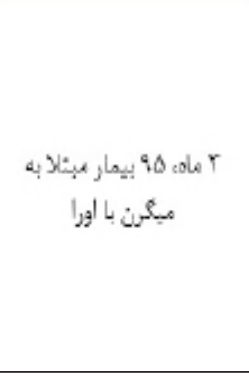 & 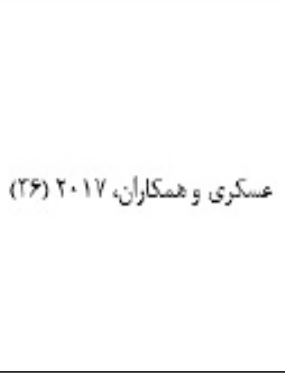 \\
\hline
\end{tabular}

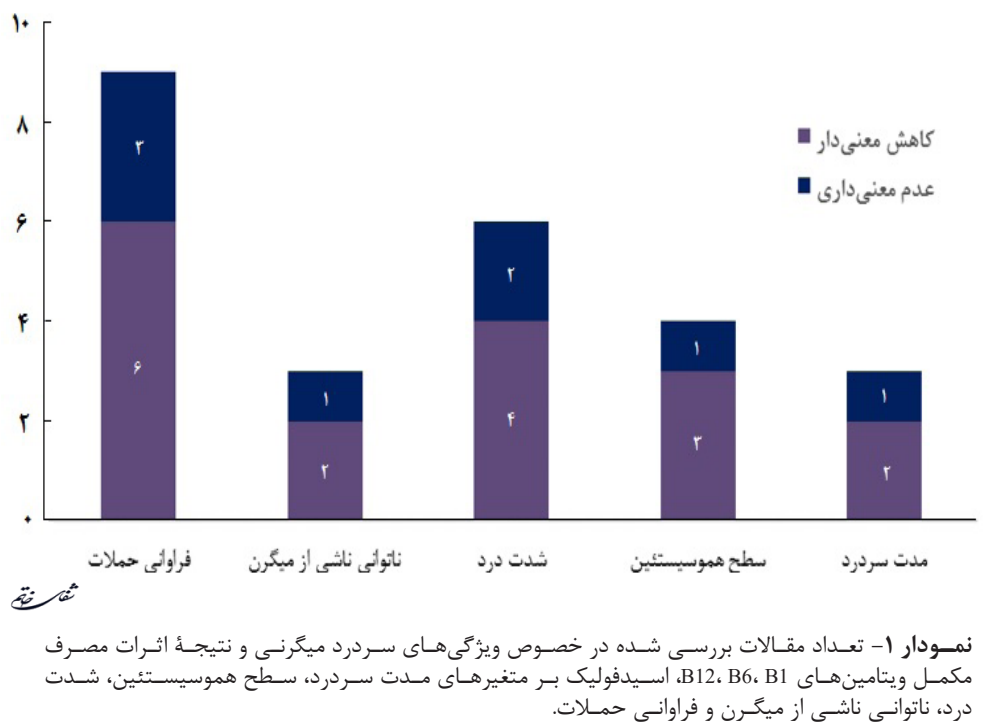




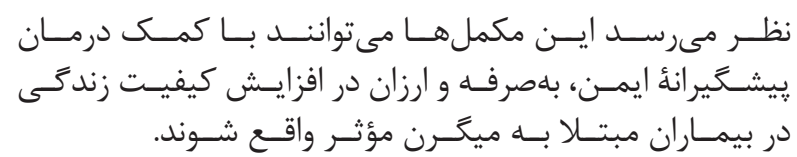

1. Faraji H, Paknahad Z, Chitsaz A. Dietary intake of thiamine in migraine patients and healthy subjects: a case-control study. Clin Nutr Res. 2018; 7(1): 40-7.

2. Menon S, Lea RA, Roy B, Hanna M, Wee S, Haupt LM, et al. Genotypes of the MTHFR C677T and MTRR A66G genes act independently to reduce migraine disability in response to vitamin supplementation. Pharmacogenet Genomics. 2012; 22(10): 741-9.

3. Shaik MM, Gan SH. Vitamin supplementation as possible prophylactic treatment against migraine with aura and menstrual migraine. Biomed Res Int. 2015; 2015. doi: 10.1155/2015/469529.

4. Farhadi Z, Alidoost S, Behzadifar M, Mohammadibakhsh R, Khodadadi N, Sepehrian R, et al. The prevalence of migraine in Iran: a systematic review and meta-analysis. Iranian Red Crescent Med J. 2016; 18(10): e40061. doi:10.5812/ircmj.40061.

5. Charles A. The pathophysiology of migraine: implications for clinical management. Lancet Neurol. 2018; 17(2): 174-82.

6. İpçioğlu OM, Özcan Ö, Gültepe M, Tekeli H, Şenol MGJTJoMS. Functional vitamin B12 deficiency represented by elevated urine methylmalonic acid levels in patients with migraine. Turk J Med Sci. 2008; 38(5): 409-14.

7. Zhang Y, Kong Q, Chen J, Li L, Wang D, Zhou JJC. International classification of headache disorders $3^{\text {rd }}$ edition beta-based field testing of vestibular migraine in China: demographic, clinical characteristics, audiometric findings and diagnosis statues. Cephalalgia. 2016; 36(3): 240-8.

8. Aurora SK, Brin MF. Chronic migraine: an update on physiology, imaging, and the mechanism of action of two available pharmacologic therapies. Headache. 2017; 57(1): 109-25.

9. Burstein R, Noseda R, Borsook D. Migraine: multiple processes, complex pathophysiology. J Neurosci. 2015; 35(17): 6619-29.

10. Slavin M, Ailani J, Reports N. A clinical approach to addressing diet with migraine patients. Curr Neurol Neurosci Rep. 2017; 17(2): 17. doi: 10.1007/s11910017-0721-6.

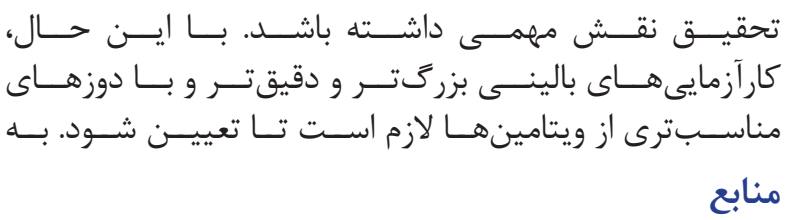

11. Gasparini CF, Sutherland HG, Griffiths LR. Studies on the pathophysiology and genetic basis of migraine. Curr Genomics. 2013; 14(5): 300-15.

12. Nattagh-Eshtivani E, Sani MA, Dahri M, Ghalichi F, Ghavami A, Arjang P, et al. The role of nutrients in the pathogenesis and treatment of migraine headaches: Review. Biomed Pharmacother. 2018; 102: 317-25.

13. Shaik MM, Tan HL, Kamal MA, Gan SH, Targets ND-D. Do folate, vitamins B6 and B12 play a role in the pathogenesis of migraine? The role of pharmacoepigenomics. CNS Neurol Disord Drug Targets. 2014; 13(5): 828-35.

14. Lea R, Colson N, Quinlan S, Macmillan J, Griffiths L. The effects of vitamin supplementation and MTHFR (C677T) genotype on homocysteine-lowering and migraine disability. Pharmacogenet Genomics. 2009; 19(6): 422-8.

15. Sadeghian H, Karimzadeh F, Jafarian M. Mechanisms Involved in pathophysiology of different types of migraines. Shefaye Khatam. 2013; 1(4): 50-4.

16. Ferraris E, Marzocchi N, Brovia D, Castellana CN, Pini L. Homocysteine levels and cardiovascular disease in migraine with aura. J Headache Pain. 2003; 4(2): 62-6.

17. Just S, Arndt K, Weiser T, Doods H. Pathophysiology of migraine: a role for neuropeptides. Drug Discovery Today: Disease Mechanisms. 2006; 3(3): 327-33.

18. Sadeghi O, Maghsoudi Z, Khorvash F, Ghiasvand R, Askari G. Assessment of pyridoxine and folate intake in migraine patients. Adv Biomed Res. 2016; 5: 47. doi: 10.4103/2277-9175.178800.

19. Sadeghi O, Nasiri M, Maghsoudi Z, Pahlavani N, Rezaie M, Askari G. Effects of pyridoxine supplementation on severity, frequency and duration of migraine attacks in migraine patients with aura: A double-blind randomized clinical trial study in Iran. Iran J Neurol. 2015; 14(2): 74-80.

20. Calik M, Aktas MS, Cecen E, Piskin IE, Ayaydın H, Ornek $\mathrm{Z}$, et al. The association between serum vitamin B 12 deficiency and tension-type headache in Turkish children. Neurological Sciences. 2018; 39(6): 1009-14.

21. Prakash S, Kumar Singh A, Rathore C, Pain F. Chronic migraine responding to intravenous thiamine: a 
report of two cases. The Journal of Head and Face Pain. 2016; 56(7): 1204-9.

22. Silaste ML, Rantala M, Sampi M, Alfthan G, Aro A, Kesäniemi Y. Polymorphisms of key enzymes in homocysteine metabolism affect diet responsiveness of plasma homocysteine in healthy women. J Nutr. 2001; 131(10): 2643-7.

23. Menon S, Nasir B, Avgan N, Ghassabian S, Oliver C, Lea $\mathrm{R}$, et al. The effect of $1 \mathrm{mg}$ folic acid supplementation on clinical outcomes in female migraine with aura patients. J Headache Pain. 2016; 17(1): 60. doi: 10.1186/ s10194-016-0652-7.

24. Maqbool MA, Aslam M, Akbar W, Iqbal Z. Biological importance of vitamins for human health: A review. J. Agric. Basic Sci. 2018; 2(3): 50-8.

25. Gabriel SE, Jaakkimainen L, Bombardier C, Carson J. Risk for serious gastrointestinal complications related to use of nonsteroidal anti-inflammatory drugs. A metaanalysis. Commentary. 1992; 116.

26. Sugano K, Tack J, Kuipers EJ, Graham DY, El-Omar EM, Miura S, et al. Kyoto global consensus report on Helicobacter pylori gastritis. Gut. 2015; 64(9): 1353-67.

27. Gasbarrini A, De AL, Fiore G, Gambrielli M, Franceschi F, Ojetti V, et al. Beneficial effects of Helicobacter pylori eradication on migraine. Hepatogastroenterology. 1998; 45(21): 765-70.

28. Seyyedmajidi M, Banikarim SA, Ardalan A, Hozhabrossadati SH, Norouzi A, Vafaeimanesh J. Helicobacter pylori and Migraine: Is Eradication of Helicobacter pylori Effective in Relief of Migraine Headache? Caspian Journal of Neurological Sciences. 2016; 2(4): 29-35.

29. Van der Kuy PH, Merkus F, Lohman J, Berg Jt, Hooymans P. Hydroxocobalamin, a nitric oxide scavenger, in the prophylaxis of migraine: an open, pilot study. International Headache Society. 2002; 22(7): 513-9.

30. Di Rosa G, Attinà S, Spanò M, Ingegneri G, Sgrò DL, Pustorino G, et al. Efficacy of folic acid in children with migraine, hyperhomocysteinemia and MTHFR polymorphisms. The Journal of Head and Face Pain. 2007; 47(9): 1342-4.

31. Oterino A, Toriello M, Valle N, Castillo J, AlonsoArranz A, Bravo Y, et al. The relationship between homocysteine and genes of folate-related enzymes in migraine patients. The Journal of Head and Face Pain. 2010; 50(1): 99-168.

32. Crider KS, Zhu J-H, Hao L, Yang Q-H, Yang TP,
Gindler J, et al. MTHFR $677 \mathrm{C} \rightarrow \mathrm{T}$ genotype is associated with folate and homocysteine concentrations in a large, population-based, double-blind trial of folic acid supplementation. Am J Clin Nutr. 2011; 93(6): 1365-72.

33. Kowa H, Yasui K, Takeshima T, Urakami K, Sakai F, Nakashima K. The homozygous C677T mutation in the methylenetetrahydrofolate reductase gene is a genetic risk factor for migraine. Am J Med Genet. 2000; 96(6): 762-4.

34. Scher AI, Terwindt GM, Verschuren WM, Kruit MC, Blom HJ, Kowa H, et al. Migraine and MTHFR C677T genotype in a population-based sample. Ann Neurol. 2006; 59(2): 372-5.

35. Menon S, Lea RA, Ingle S, Sutherland M, Wee S, Haupt LM, et al. Effects of dietary folate intake on migraine disability and frequency. The Journal of Head and Face Pain. 2015; 55(2): 301-9.

36. Askari G, Nasiri M, Mozaffari-Khosravi H, Rezaie M, Bagheri-Bidakhavidi M, Sadeghi O. The effects of folic acid and pyridoxine supplementation on characteristics of migraine attacks in migraine patients with aura: A double-blind, randomized placebocontrolled, clinical trial. Nutrition. 2017; 38: 74-9.

37. Liverman C, Brown J, Sandhir R, Klein R, McCarson $\mathrm{K}$, Berman N. Oestrogen increases nociception through ERK activation in the trigeminal ganglion: evidence for a peripheral mechanism of allodynia. International Headache Society. 2009; 29(5): 520-31.

38. Tully DB, Allgood VE, Cidlowski JA. Modulation of steroid receptor-mediated gene expression by vitamin B6. FASEB J. 1994;8(3): 343-9.

39. Dhillon KS, Singh J, Lyall J. A new horizon into the pathobiology, etiology and treatment of migraine. Med Hypotheses. 2011; 77(1): 147-51.

40. Bâ A. Metabolic and structural role of thiamine in nervous tissues. Cell Mol Neurobiol. 2008; 28(7): 923-31.

41. Mkrtchyan G, Aleshin V, Parkhomenko Y, Kaehne T, Di Salvo ML, Parroni A, et al. Molecular mechanisms of the non-coenzyme action of thiamin in brain: biochemical, structural and pathway analysis. Scientific Reports. 2015; 5: 12583.

42. Schoenen J, Jacquy J, Lenaerts M. Effectiveness of high-dose riboflavin in migraine prophylaxis A randomized controlled trial. Neurology. 1998; 50(2): 466-70.

43. Mahan LK, Escott-Stump S, Raymond JL, Krause MV. Krause's food \& the nutrition care process. Elsevier Health Sciences. 2012. 